\title{
Miniaturized E5a/E1 Antenna Array for Robust GNSS Navigation
}

\author{
S. Caizzone, IEEE Member
}

\begin{abstract}
This paper discusses the design and manufacturing of a miniaturized $2 \times 2$ stacked patch antenna array, operating at E5a and E1 Galileo bands (i.e. respectively at central frequencies of 1176 and $1575 \mathrm{MHz}$ ), for robust navigation applications. The $2 \times 2$ array configuration has a total dimension of $100 \mathrm{~mm} \times 100$ $\mathrm{mm}$, hence enabling its use in mobile applications. Due to the tight placement of nearby elements, mutual coupling problems arise. The reduction of the electromagnetic interaction among the elements is pursued by means of a metallic fence.
\end{abstract}

Index Terms-GNSS, Array, mutual coupling, compact antenna

\section{INTRODUCTION}

Global Navigation Satellite Systems (GNSS) are growing in number and applications. In particular, the upcoming of full functionalities for the European Galileo GNSS promises enhanced possibilities in terms of accurate navigation [1].

Such enhancement in terms of availability and accuracy of navigation signals is triggering the development of new applications: some examples are for instance precise synchronization, precise timing, as well as precise and robust navigation. Different groups all over the world have therefore started intensive research in order to develop robust, antijamming and anti-spoofing GNSS receivers [2], [3]. The use of arrays of antennas and ad-hoc algorithms enables in fact to steer the beam towards the satellites and place nulls towards the jammer/spoofer direction [4].

Although they provide extremely interesting performance, most of the current systems are far too big in size to be possibly mounted on a vehicle and hence used outside of research laboratories.

As a matter of fact, in order to pursue a real-life applicability of such systems, the dimensions of both the antenna arrays and the receiver have to be drastically reduced, up to dimensions compatible with mobile applications: both automotive and aeronautical sectors have e.g. very strict requirements for the placement of GNSS antennas (see e.g. ARINC specifications for aircraft antennas [5]). The need is therefore, from the antenna side, to develop miniaturized high-performance arrays, with reduced footprints, though maintaining as much as possible the capabilities (i.e. gain and bandwidth) of larger arrays.

The quest for small antennas satisfying both gain and bandwidth requirements has been an active topic of research over the last decades. Several miniaturization techniques have been proposed (for a wide review see [6]), with the use of materials having an high dielectric constant (DK) being one of the most common [7].

S. Caizzone is with the Institute of Communications and Navigation, German Aerospace Center (DLR), Oberpfaffenhofen, Germany. E-mail: stefano.caizzone@dlr.de.
Moreover, in order to have a compact system, not only the single antenna, but a total footprint miniaturization has to be achieved. The array size has thus to shrink, meaning that the single antennas will be electrically closer to each other. Such aim is by no means easily achieved, as higher degrees of miniaturization also imply increased mutual coupling and more difficult antenna matching [9]. Various efforts to solve this problem are found in literature, e.g. by means of adding a decoupling and matching network [10], [11] able to decouple the coupled patterns of the antennas, or by using, for instance, split ring resonators (SRRs) to stop the coupling between the elements [12]. These techniques are, however, manufacturingintensive and considerably increase the cost of the system.

The present work focuses instead on a solution employing commercial off the shelf (COTS) substrate materials and an easier manufacturing, leading to lower costs, both for the single antennas and for the mutual coupling reduction mechanism. From a technical point of view, the focus will be the development of a miniaturized dual band antenna array for the E1 and E5a bands of Galileo, i.e. having a $3 \mathrm{~dB}$ gain minimum bandwidth respectively of 32 and $25 \mathrm{MHz}$. The size of the $2 \times 2$ array shall be limited to $10 \times 10 \mathrm{~cm}^{2}$. Due to the compactness of the solution, mutual coupling between the elements will arise: it is however required to minimize the loss of the degrees of freedom (DOF) for the array signal processing, which translates therefore into the need of mutual coupling minimization. This will be achieved by means of metallic fences among the single antennas, in order to limit the electromagnetic interaction between them. The improvement provided by the metallic fences will be evaluated in terms of mutual S-Parameters and array eigenmode radiation efficiencies, in order to provide a complete insight into the various mechanisms of coupling.

The paper is divided as follows: Sec. II will show the design of the $2 \times 2$ array, while Sec. III will show the measurement results. Conclusions will be drawn in Sec. IV.

\section{ARRAY DESIGN}

The antennas have been designed as dual stacked patches with proximity coupled feeding (stackup in Fig. 1).

A COTS substrate material (ROGERS RO 6010) with DK 10 has been chosen for pursuing miniaturization. The single antennas will have a confined substrate extension and will therefore act as single blocks in the miniaturized $2 \times 2$ array, with overall size of $100 \mathrm{~mm}$ and mutual distance of 50 $\mathrm{mm}$ between the antennas, i.e. $\sim \lambda / 5$ at E5a band and $\sim \lambda / 4$ at E1 band (Fig. 2-a). 


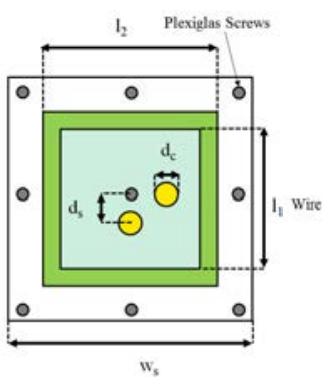

a)

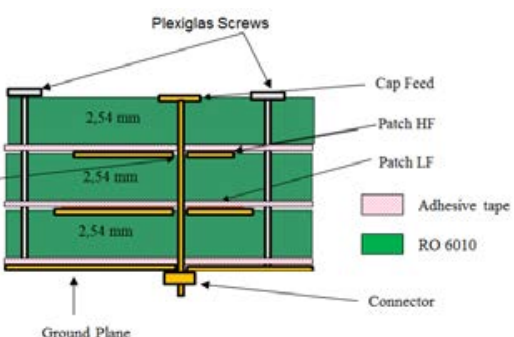

b)
Figure 1. Model of the antenna: a) top view; b) stackup

Although the antennas do not share a common substrate and therefore surface waves are diminished, the extremely small mutual distance causes nevertheless mutual coupling between the elements (Fig. 3), implying impedance mismatch and pattern distortion as expected from theory [9]. In order to center the maximum gain at E5a and E1 central frequencies, the sizes of the microstrip patches had to be adapted, changing slightly the dimensions with respect to those used for the single antenna: this is particularly true at E5a band, where the coupling is stronger and where the patch size must therefore be adapted to $l_{2}=33.9 \mathrm{~mm}$ (in contrast to $l_{2}=34.3 \mathrm{~mm}$ required for the single antenna). Fig. 3 shows the S-parameters versus frequency for this configuration.

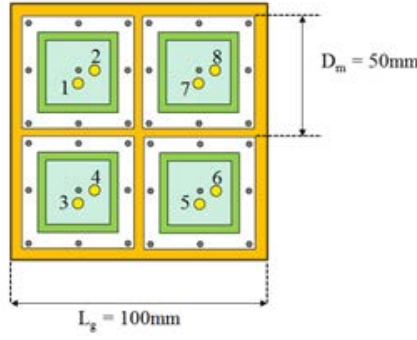

a)

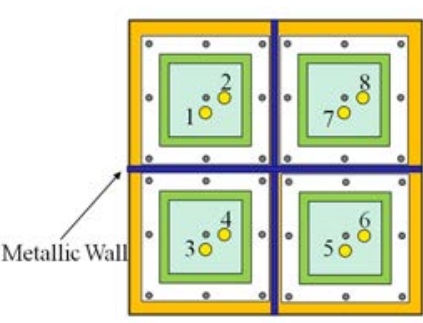

b)
Figure 2. Geometrical layout of the miniaturized $2 \times 2$ array without fences (a) and with fences (b)

It can be observed in Fig. 3-a that a strong mutual coupling is present, as also demonstrated by the high level (around $5 \mathrm{~dB}$ ) of $S_{13}$, i.e. among adjacent copolarized probes ${ }^{1}$. This situation is not optimal, because of the coupling of power from one port to the others, causing more losses and more signal correlation.

In order to limit the mutual coupling between the antennas in the array, a further technique is proposed: the antennas can be separated by a metallic wall (also called "fence"), helping in avoiding power coupling [14] from one antenna to the other (Fig. 2-b).

${ }^{1}$ For the naming of ports in the array, please refer to Fig. 2

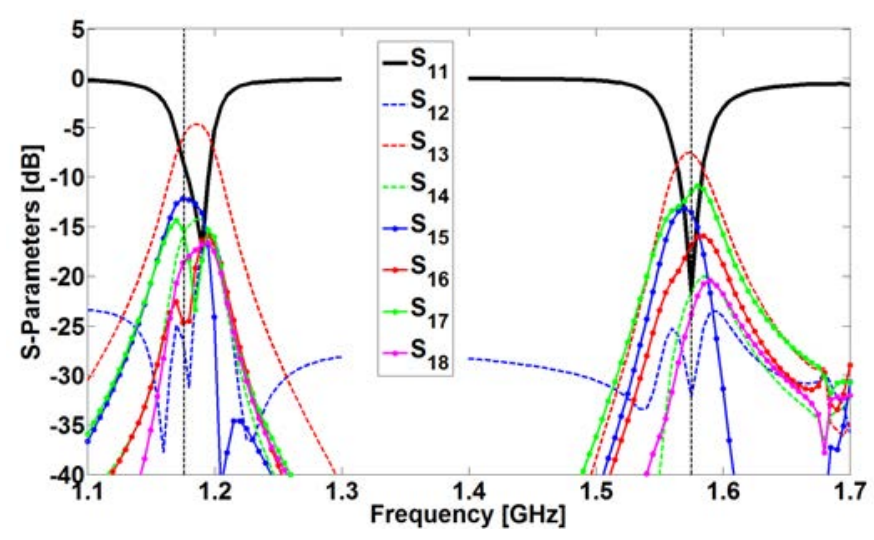

Figure 3. S- Parameters for the optimized $2 \times 2$ array (embedded case (antenna 1 fed, others load-terminated)). The geometrical dimensions are (sizes in $[\mathrm{mm}]): l_{1}=26.4, l_{2}=33.9, d_{c}=2, d_{s}=7$

Such wall constitutes an electromagnetic shield for the fields coupling from one antenna to the other and thus enables to decrease the mutual coupling between the elements, as shown in Fig. 4. In this case, the highest coupling term, i.e. $S_{13}$ is below $-10 \mathrm{~dB}$ at both frequencies and hence substantially lower with respect to the former case.

Both cases (with and without metallic wall) have been tested in real life and the results of the manufactured prototypes are shown in the next Section.

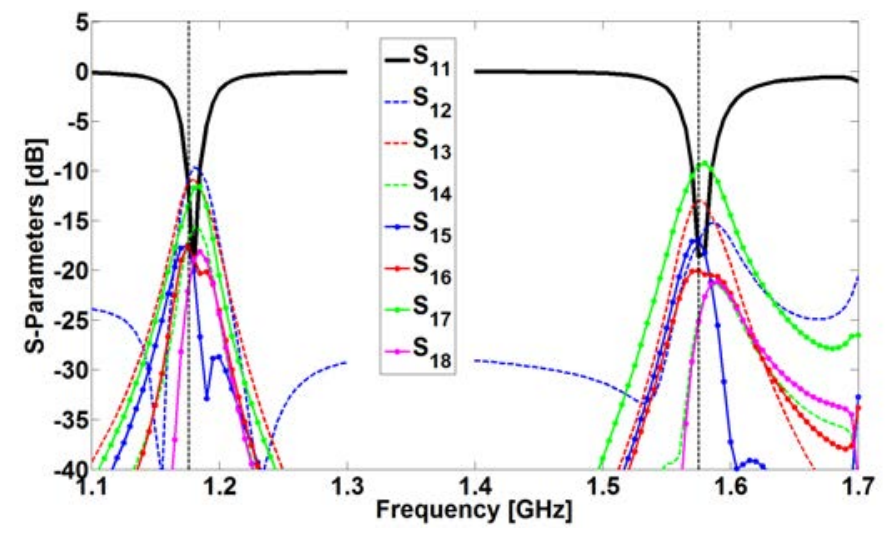

Figure 4. S- Parameters for the optimized $2 \times 2$ array with metallic wall (embedded case (antenna 1 fed, others load-terminated))

\section{MEASUREMENT}

The designed antenna arrays were manufactured in-house and then measured in a Satimo Starlab near-field antenna measurement facility (Fig. 5). The S-Parameters of the antennas without hybrids have been measured at first. All SParameters have been measured and a remarkable agreement with simulations has been found. Fig. 6 shows $S_{11}$ and $S_{13}$ (i.e. the strongest mutual coupling coefficient) for both array configurations.

A remarkable decrease in $S_{13}$ is visible for the case with fence, clearly improving the independency of one port from the other. The measured RHCP and LHCP gains at boresight versus frequency for the embedded antennas (both with and without fences) are shown in Fig. 7. 


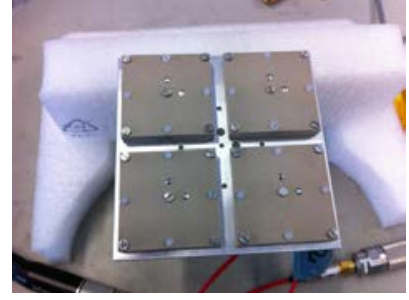

a)

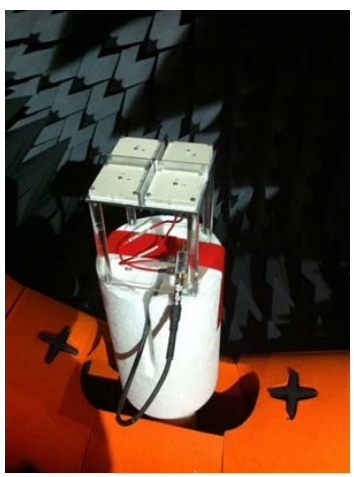

b)
Figure 5. Manufactured prototype for the miniaturized 2x2 array (a) and array during measurement in the CTR antenna measurement facility (b).

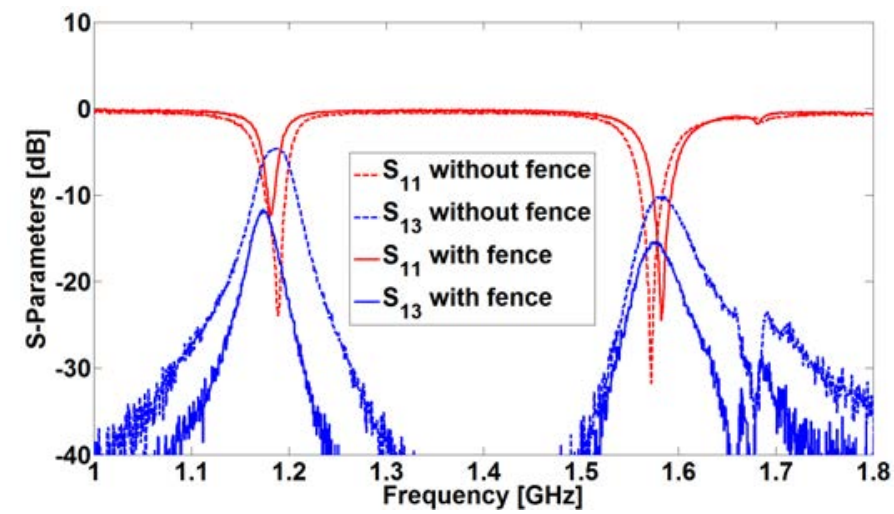

Figure 6. S- Parameters for the optimized $2 \times 2$ array with and without metallic wall (fence).

Good results are found in both cases, with a clear dual-band behaviour as well as a $3 \mathrm{~dB}$ gain bandwidth of respectively 35 and $60 \mathrm{MHz}$ for the array with metallic wall and of 55 and 70 $\mathrm{MHz}$ without the wall, largely complying with specification in both cases.

Evaluating mutual coupling through the S-Parameters provides however only a partial vision of the phenomenon, as it does not yield any explanation, for instance, about the 3D spatial distribution and interaction of the fields. In order to understand more thoroughly the positive effect of the fence on the array behaviour, additional performance parameters for compact arrays will therefore be discussed and used to compare the two proposed layouts.

One suitable parameter for such comparison is the radiation efficiency of the eigenmodes of the array [15].

The radiation characteristics of a $\mathrm{N}$-element array can be in fact analyzed through the use of $\mathrm{N}$ radiation eigenmodes [15], analytically obtained by decomposing the radiation matrix $\widetilde{H}=\widetilde{I}-\widetilde{S}^{H} \widetilde{S}$ through an eigenvalue decomposition (EVD) into a diagonal matrix of eigenvalues $\widetilde{\Lambda}=$ $\operatorname{diag}\left\{\lambda_{1}, \lambda_{2}, \ldots, \lambda_{N}\right\}$ and a matrix of orthogonal eigenvectors $\widetilde{Q}:$

$$
\widetilde{H}=\widetilde{Q} \widetilde{\Lambda} \widetilde{Q}^{H}
$$

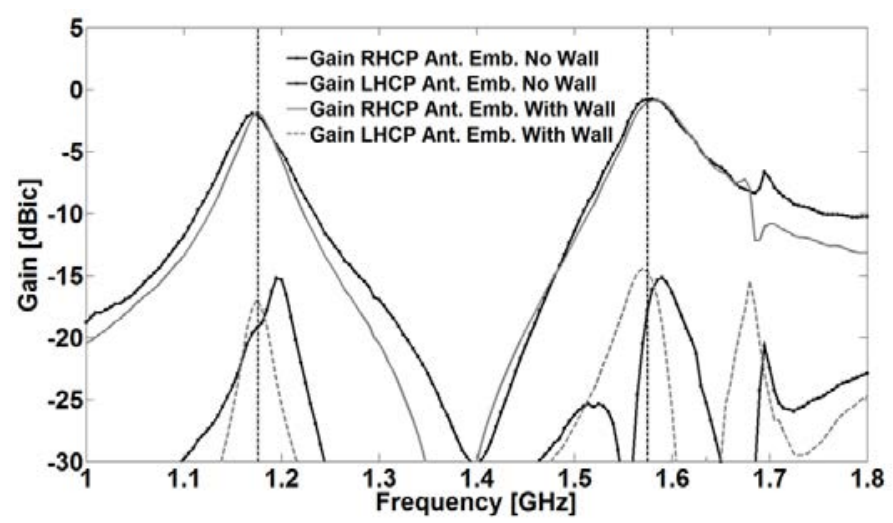

Figure 7. Measured gain, at boresight and versus frequency, for the antenna embedded in the array either without or with the metallic wall

The obtained eigenmodes are orthogonal, in the sense that their beam overlap integral [16] vanishes for distinct beams: therefore, they can be considered as independent degrees of freedom (DOFs) in the signal processing level (e.g. for DOA estimation or null steering). The eigenmodes differentiate in their spatial distribution (Fig. 8) and therefore provide useful information on the radiation capability of the array in the different directions.

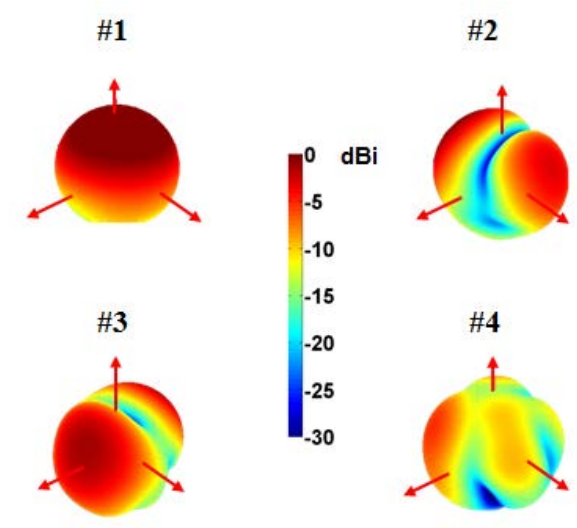

Figure 8. Measured 3D eigenmodes gain for the array with metallic wall, at E1 central frequency $f=1575 \mathrm{MHz}$

The eigenvalues are related to the eigenefficiencies (or radiation efficiencies) of the eigenmodes, i.e. "the proportion of power in the eigenmode that is actually radiated into the farfield" [15]. A deeper investigation on the actual eigenefficiencies of the proposed arrays will therefore provide an insight into the applicability of each mode (and hence on the radiating capability of the array in the mode main directions): low eigenefficiencies, as often encountered in compact arrays with high coupling [11], are for instance associated with the practical unusability of the corresponding mode (expressed by a very low gain of the mode) and therefore with a factual loss in the available degrees of freedom for signal processing, limiting the array capabilities, e.g. in terms of number of suppressed jammers.

The two array layouts shown previously have therefore been tested to collect the required eigenefficiencies directly 
from farfield measurements. In order to excite the different eigenmodes in measurement, each array has been successively connected to different configurations of a network of COTS power couplers able to provide the required amplitude and phase values for the excitation of the actual eigenmode ${ }^{2}$. The measured 3D patterns of the eigenmodes at E1 central frequency for the array with metallic wall are shown in Fig. 8 , where the typical shape of higher order modes (with more lobes) is clearly visible.

Tab. I provides a comparison between the measured radiation efficiencies and maximum RHCP gains of the eigenmodes in both array configurations (with and without metallic wall) at the central frequencies of E5a and E1 bands.

\begin{tabular}{|c|c|c|c|c|}
\hline & No Wall, E5a & Wall, E5a & No wall, E1 & Wall, E1 \\
\hline \hline Mode \#1 & $-2.8 / 3.5$ & $-3.2 / 3.1$ & $-3.3 / 3.7$ & $-3.8 / 3.6$ \\
\hline Mode \#2 & $-8.8 /-3.8$ & $-8.2 /-1.7$ & $-6.9 /-0.4$ & $-6.7 /-0.6$ \\
\hline Mode \#3 & $-8.1 /-3.2$ & $-7.6 /-1.2$ & $-6.4 /-0.5$ & $-6.3 /-0.7$ \\
\hline Mode \#4 & $-16.9 /-13.6$ & $-13.0 /-6.0$ & $-11.0 /-5.0$ & $-10.7 /-4.8$ \\
\hline
\end{tabular}

Table I

MEASURED EIGENMODE RADIATION EFFICIENCY/MAXIMUM RHCP GAIN FOR THE ARRAYS WITH AND WITHOUT METALLIC WALL (VALUES IN DB)

It is clearly visible that, at E5a, better radiation efficiencies are obtained for higher modes in the configuration with wall (e.g. ca. $0.5 \mathrm{~dB}$ improvement for modes 2 and 3 and $3.9 \mathrm{~dB}$ improvement for mode 4): this improvement, combined with the more pronounced squint of the patterns in the configuration with walls, causes then a substantial enhancement of the maximum RHCP gain for such modes (Tab. I), with around $2 \mathrm{~dB}$ improvement for modes 2 and 3 and more than $7 \mathrm{~dB}$ improvement for mode 4 . Mode 1 , on the other hand, does not profit from the presence of the fence: as already noticed, the presence of metallic walls causes the embedded patterns to be actually slightly squinted from zenith, due to the scattering of the metallic wall itself, and therefore the maximum gain of Mode 1 (which indeed points towards zenith) is lower for the wall case than for the configuration without the wall. At E1 band, moreover, where the coupling is less critical also without the metallic wall, very similar results are obtained for both array configurations.

The obtained results show therefore how, by using the metallic wall and hence reducing the mutual coupling between the antennas in the array, it is possible to obtain a valuable improvement in the radiation efficiencies of the higher order modes. Having higher modes with better efficiency is extremely valuable, as it provides the algorithms in the signal processing chain with more balanced (and therefore more exploitable) degrees of freedom, with a better performance mostly at low elevations from horizon, i.e. where it is most needed to counteract ground-based interferers.

\section{CONCLUSION}

The present paper has shown the design of a compact dual band antenna array for GNSS applications. The compact array having a total dimension of only $10 \times 10 \mathrm{~cm}^{2}$, has been

\footnotetext{
${ }^{2}$ The modes have the following eigenvectors, i.e. phase and amplitude

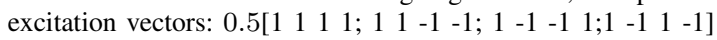

analyzed in two different configurations, with and without the mutual coupling reduction effect provided by a metallic wall placed in between the antennas. Measurements show the beneficial effect of the metallic wall, both in terms of reduced mutual S parameters and improved radiation efficiency of the eigenmodes of the array. The proposed layout is therefore best suited as miniaturized dual band solution to be connected to the array signal processing unit for robust GNSS navigation.

\section{ACKNOWLEDGMENT}

The author would like to thank Mr. Elmarissi and Mr. Buchner for the precious support during the manufacturing and measurement phases, as well as Dr. Dreher and Prof. M. A. Hein for fruitful discussion. The research, whose results are reported in this paper, was performed in the frame of the KOSERNA project, co-funded by the Bavarian Ministry of Economic Affairs and Media, Energy and Technology. This support is greatly acknowledged.

\section{REFERENCES}

[1] C. J. Hegarty, E. Chatre, "Evolution of the Global Navigation Satellite Systems (GNSS)", Proc. of the IEEE, vol. 96, no. 12, pp. 1902-1917, Dec. 2008

[2] A. Hornbostel, A. Konovaltsev, H. Denks, F. Antreich, "Simulation of Multi-Element Antenna Systems for Navigation Applications", IEEE Sys. Journal, vol. 2, no. 1, pp. 7-20, Mar. 2008

[3] R. L. Fante, J. J. Vacarro, "Cancellation of Jammers and Jammers Multipath in a GPS Receiver", IEEE Aerosp. and Electronic Syst. Mag., vol. 13 , no. 11 , pp. $25-28$, Nov. 1998

[4] M. V. T. Heckler, M. Cuntz, A. Konovaltsev, L. Greda, A. Dreher, M. Meurer , "Development of robust safety-of-life navigation receivers", IEEE Trans. Microw. Theory Tech., vol. 59, no. 4, pp. 998-1005, Apr. 2011

[5] ARINC 743 Specification, downloadable at www.aviation-ia.com

[6] J. Volakis, C.-C. Chen, K. Fujimoto, "Small Antennas: Miniaturization Techniques and Applications", Mc Graw Hill Professional, 2009

[7] L. Yue, C.-C. chen, D. Psychoudakis, J. L. Volakis, "Miniaturized 1" Dual-Band GPS Antenna Element", Proc. of the 2010 IEEE Antennas Propag. Soc. Symp. (APSURSI), Toronto, Jul. 2010

[8] H.-M. Chen, Y.-F. Lin, C.-H. Chen, C.-Y. Pan, Y.-S. Cai, "Miniature Folded Patch GPS Antenna for Vehicle Communication Devices", IEEE Trans. Antennas Propag., vol. 63, no. 5, 1891-1898, May 2015

[9] S. Caizzone, A. Dreher, "An Uncoupled Rematching Technique for Coupled Receive Arrays", Proc. of the 2014 German Microwave Conf. (GEMIC), Aachen (Germany), Mar. 2014

[10] S. Irteza, N. Murtaza, S. Caizzone, R. Stephan, M. A. Hein , "Compact Planar L-Band Antenna Arrays with Optimal Diversity Performance", Proc. of the 2011 IEEE Conf. Antennas and Propag. in Wireless Comm. $(A P W C)$, Torino (Italy), Sept. 2011

[11] M. Ibraheam, S. Irteza, S. Caizzone, A. Dreher, R. Stephan, M. A. Hein "Compact Dual Band Dual Polarized Antenna Array for Robust Satellite Navigation Receivers", Proc. of European Conf. on Antennas Propag. (EUCAP 2015), Lisbon (Portugal), Apr. 2015

[12] A.A. Gheethan, P. A. Herzig, G. Mumcu, "Compact 2x2 Coupled Double Loop GPS Antenna Array Loaded with Broadside Coupled Split Ring Resonators", IEEE Trans. Antennas Propag., vol. 61, no. 6, pp. 30003008, Jun. 2013

[13] Z. Wang, S. Fang, S. Fu, S. Lu, "Dual-Band Probe-Fed Stacked Patch Antenna for GNSS Applications", IEEE Antennas Wireless Propag. Letters, vol. 8, p. 100-103, Jan. 2009

[14] R. J. Mailloux, "Reduction of Mutual Coupling using Perfectly Conducting Fences", IEEE Trans. Antennas Propag. , vol. 19, no. 2, pp. 166-173, Mar. 1971

[15] C. Volmer, J. Weber, R. Stephan, K. Blau, M. A. Hein, "An EigenAnalysis of Compact Antenna Arrays and its Application to Port Decoupling", IEEE Trans. Antennas Propag., vol. 56, no. 2, pp. 360-371, Feb. 2008

[16] S. Stein, "On Cross Coupling in Multiple-Beam Antennas", IRE Trans. Antennas Propag., vol. 10, no. 5, pp. 548-557, Sep. 1962 